Chirurg 2011 $\cdot 82: 757-758$

DOI 10.1007/s00104-011-2091-y

Online publiziert: 25. August 2011

(c) Springer-Verlag 2011

\author{
G. Germann \\ ETHIANUM - Klinik für Plastische \& Rekonstruktive Chirurgie, \\ Präventive und Ästhetische Medizin am Universitätsklinikum Heidelberg
}

\section{Ästhetische Chirurgie}

\section{Wiederherstellung des Körperbildes zur Erhöhung der Lebensqualität}

Über viele Jahrzehnte stellte es für die plastische Chirurgie eine große Herausforderung dar, ihre fachlichen Inhalte als unverwechselbare Entität im chirurgischen Fächerkanon darzustellen. Obwohl die in der plastischen Chirurge entwickelten rekonstruktiven Techniken für alle Fächer neue therapeutische Optionen boten und gleichzeitig die Begehrlichkeiten anderer Fachbereiche weckten, wurde das Fach selbst innerhalb des chirurgischen Kollegenkreises häufig ausschließlich gleichgesetzt mit ästhetischer Chirurgie, deren Eingriffe nicht, wie in vielen anderen Fachbereichen, primär auf die Wiederherstellung nach Unfall oder der Behandlung maligner Tumoren oder anderer lebensbedrohlicher Erkrankungen dienten.

Erst in den letzten Jahren, mit der Neudefinition des Gebietes Chirurgie und dem engeren Zusammenschluss der beteiligten wissenschaftlichen Fachgesellschaften, erlebte die plastische Chirurgie mit all ihren Facetten eine höhere Akzeptanz, nicht zuletzt bedingt durch viele Felder der Kooperationen, bei denen erst die komplexen plastisch-chirurgischen Eingriffe neue Dimensionen der chirurgischen Therapie ermöglichten.

Trotz einer differenzierten Darstellung des gesamten Leistungsspektrums des Fachgebietes hält sich hartnäckig sowohl bei Kollegen als auch in der Öffentlichkeit, auch geschürt durch entsprechende Medienberichte, das Image der plastischen Chirurgie als ästhetischer Erfüllungsgehilfe eines von der modernen Mediengesellschaft vorgegebenen Schönheitsideals. Dabei werden teilweise in entwürdigen- der medialer Instrumentalisierung von Patienten immer wieder Kollegen herangezogen, die nicht einmal plastische Chirurgen sind. Diese lassen sich dabei nicht ungern zur Bedienung des Klischees der reinen "Lifestyle-Chirurgie“ in "Soapähnlichen“ Genres inszenieren und erzeugen mit permanenter Medienpräsenz eben dieses verzerrte Bild der plastischen Chirurgie.

Umso erfreulicher ist es für uns plastische und ästhetische Chirurgen, dass nun der Herausgeber von Der Chirurg mit der Bitte an mich herantrat, das Leitthema „Ästhetische Chirurgie“ für diese Facharztzeitschrift zu gestalten. Dabei darf nicht unberücksichtigt bleiben, dass sich die plastischen Chirurgen in Der Chirurg seit Jahren sehr engagieren und in der Spitzengruppe der publizierten Arbeiten liegen. Nicht zuletzt dieses Engagement hat dazu geführt, dass viele Vorurteile und Fehleinschätzungen, gerade im rekonstruktiven Bereich, beseitigt werden konnten. Nicht zuletzt deshalb haben alle angesprochenen Autoren trotz eines engen Zeitfensters sofort große Bereitschaft gezeigt, das Leitthema „Ästhetische Chirurgie“ in seiner ganzen Bandbreite aufzubereiten.

Betrachtet man die WHO-Definition von Gesundheit, so fällt auf, dass es hier nicht mehr auf die „absence of illness“, sondern auch auf „the presence of wellbeing " ankommt. Für die ästhetische Chirurgie bedeutet dies, dass bei seriöser Indikationsstellung ein Mehr an Lebensqualität für die Betroffenen erreicht werden kann und muss. Damit steht die plastische Chirurgie durchaus nicht alleine. Eingrif- fe zur Erhöhung der Lebensqualität gibt es auch in anderen Fächern. So stellen beispielsweise nicht alle Leistenhernien zwingend eine vitale Indikation zur Operation dar und auch in der Orthopädie/ Unfallchirurgie gibt es Indikationen, bei denen z. B. der Gelenkersatz vornehmlich der Beseitigungen von Schmerzen und damit der Verbesserung der Lebensqualität dient.

\section{Ohne rekonstruktive Grundlagen ist keine seriöse ästhetische Chirurgie möglich}

Natürlich bewegt sich dabei die ästhetische Chirurgie mehr als andere Fächer in einer indikatorischen Grauzone zwischen rein medizinisch notwendiger und wunscherfüllender Chirurgie. Es darf nicht vergessen werden, dass es sich hier auch um einen „Wachstumsmarkt“ handelt, auf den viele Kollegen drängen, die in keiner Weise dafür qualifiziert sind. Leider unterstützt der Gesetzgeber diese Tendenzen auch durch Urteile, wie unlängst durch das Bundesverfassungsgericht, dass die Ausübung ästhetischer Eingriffe auch bei fehlender fachlicher Qualifikation alleine „qua approbatione“ gerechtfertigt sei. Dies stellte zunächst die Facharztweiterbildung in plastischer und ästhetischer Chirurgie in Frage. Mittlerweile hat aber die Bundesärztekammer in der Novelle der Musterberufsordnung Klarheit geschafft und formuliert:

Eine gewissenhafte Ausübung des Berufs erfordert insbesondere die notwendige fachliche Qualifikation und die Beach- 
tung des anerkannten Standes der medizinischen Erkenntnisse.

Diese Formulierung macht eindeutig klar, dass Ärzte, die ohne hinreichende Qualifikation beispielsweise ästhetisch-chirurgische Operationen durchführen, berufswidrig handeln. Dennoch ist der Patient, häufig nicht in der Lage, das Dickicht der Facharztdefinitionen, Zusatzbezeichnungen und phantasievoller Eigenkreationen von Spezialistenbezeichnungen zu durchschauen. Er wird so leider immer wieder zum Opfer nicht ausgebildeter Kollegen, die in Wochenendseminaren versuchen, die Facharztweiterbildung zu ersetzen oder die ästhetische Chirurgie im Do-it-yourself-Verfahren erlernen.

Gerade in einem Zeitalter des sich ständig erhöhenden Wettbewerbsdruck, darf sich die ästhetische Chirurgie nicht zum Erfüllungsgehilfen medial vorgegebener Schönheitsideale machen und jeden Patientenwunsch, ungeachtet einer vorliegenden Indikation, erfüllen.

Dennoch wird es Fälle geben, in denen Indikationen „weiter gefasst“ werden, als es eigentlich angebracht wäre, um Patienten nicht zu verlieren. Dabei darf aber nicht übersehen werden, dass der Ruf anerkannter ästhetische Chirurgen vor allem auf seriöser Beratung, auch mit Verweigerung von Operationswünschen, und technisch akkurater Ausführung der Eingriffe mit geringen Komplikationsraten und langfristig guten Ergebnissen beruht. Um ein Operationsverfahren individuell auf die Bedürfnisse des Patienten abzustimmen, bedarf es häufig intensiver, auch mehrfacher Beratungen.

Die ästhetische Chirurgie besitzt ein großes Potenzial das Lebensgefühl von Patienten zu verbessern. Dies wird vor allem in den Bereichen deutlich, die per se nicht als ästhetische Chirurgie eingeordnet werden, wie z. B. die Korrektur abstehender Ohren oder die Korrektur von Fehlstellungen nach Nasenbeinfrakturen. Hier stimmt auch die breite Öffentlichkeit sofort zu, dass solche Zustände korrigiert werden sollten, um den Betroffenen Hänseleien oder die ,aufmerksamen“ Blicke der Umwelt zu ersparen. Dies trifft auch für Fehlbildungen der weiblichen Brust zu, weil natürlich niemand bestreiten möchte, dass es zu erheblichen psy- chischen Störungen kommen kann, wenn massive Asymmetrien der weiblichen Brust in der Adoleszenzphase vorliegen.

In der öffentlichen Diskussion über Rechtfertigung und Stellenwert der ästhetischen Chirurgie beispielweise bei Altersveränderungen zeigen sich zwischen westlichen Ländern deutliche Unterschiede. Schnell ist hier von „man soll doch in Würde altern“ oder „Lifestyle-Chirurgie“ die Rede. Neuere Ergebnisse einer ForsaUmfrage zeigen jedoch, dass in der Altersgruppe unter 6o Jahren mehr als 70\% der Befragten ästhetische Chirurgie für einen normalen Bestandteil der Gesellschaft halten, dass sie zu mehr Wohlbefinden der Menschen beiträgt und moralisch nicht zu verurteilen ist. Diese Zahlen deuten eine gewisse Umkehr in der Haltung der Bevölkerung in Deutschland an. Wurde z. B. in Frankreich, Italien oder Spanien die ästhetische Chirurgie offen im sozialen Umfeld diskutiert, fand ästhetische Chirurgie in Deutschland eher heimlich statt. Dabei ist es mittlerweile unbestritten, dass plastische Chirurgie Lebensqualität und das Selbstwertgefühl hebt und viele Menschen mit neuem Selbstvertrauen in ihr Leben starten, wie auch eine kürzlich veröffentlichte psychologische Studie aus der Universität Leipzig zeigen konnte.

Ein Paradigmenwechsel hat dazu geführt, dass heute das Ziel der Eingriffe nicht mehr die bloße Straffungsoperation ist, die in der Vergangenheit häufig zu den typischen Aspekten des „ästhetisch operiert aussehen" führte - was leider gerade in den USA häufig zur negativen Publicity der Ergebnissen führt, sondern die Optimierung einer Ausgangssituation unter Beibehaltung der grundsätzlichen Charakteristika.

In den Beiträgen dieses Leitthemenheftes haben namhafte Kollegen sich der Aufgabe gewidmet, jeweils einen Teilaspekt der ästhetischen Chirurgie in diesem Sinne zu beleuchten und einen Einblick in die derzeit gebräuchlichen Techniken zu geben. Als typisches Beispiel für die Überlappung ästhetischer Chirurgie und rekonstruktiver Chirurgie wurde bewusst die Wiederherstellung der weiblichen Brust aufgenommen. Die Rekonstruktion der weiblichen Brust dient der Wiederherstellung des Körperbildes und damit der Wiederherstellung des Selbstwertgefühls der betroffenen Frau. Die Wichtigkeit dieser Operation wird von keinem Kollegen, Kassenvertreter oder gesundheitspolitisch Tätigen bestritten, ist aber dennoch ein Beispiel dafür, dass eine eigentlich der ästhetischen Wiederherstellung des Körperbildes dienende Operation zu einer signifikanten Verbesserung der seelischen Befindlichkeit führt und deshalb als ideales Beispiel für die These gelten darf, dass ohne ästhetische Prinzipien keine moderne Rekonstruktion möglich ist, dass aber ohne Beherrschung der rekonstruktiven Grundlagen seriöse ästhetische Chirurgie nicht möglich ist.

In diesem Sinne wünsche ich Ihnen viel Spaß beim Lesen der Beiträge.

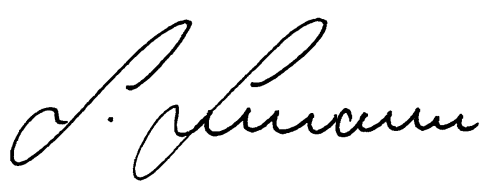

Prof. Dr. G. Germann

\section{Korrespondenzadresse \\ Prof. Dr. G. Germann \\ ETHIANUM - \\ Klinik für Plastische \& Rekonstruktive Chirurgie, Präventive und Ästhetische Medizin am Universitätsklinikum Heidelberg, Voßstr. 6, 69115 Heidelberg \\ Guenter.Germann@ethianum.de}

